\title{
Study of the muon content of high-energy air showers with KASCADE-Grande
}

\author{
J.C. Arteaga-Velázquez ${ }^{1, *}$, D. Rivera-Rangel ${ }^{1, * *}$, W.D. Apel $^{2}, K$. Bekk ${ }^{2}, M$. Bertaina $^{3}, J$. Blümer $^{2,4,14}, H$. Bozdog $^{2}, I . M$. \\ Brancus $^{5}, E$. Cantoni ${ }^{3,6,15}, A$. Chiavassa ${ }^{3}, F$. Cossavella ${ }^{4,16}, K$. Daumiller ${ }^{2}, V$. de Souza ${ }^{7}, F$. Di Pierro ${ }^{3}, P$. Doll $^{2}, R$.

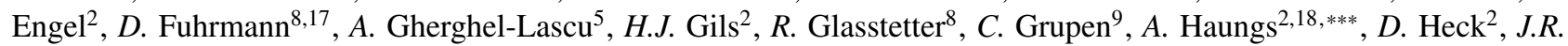

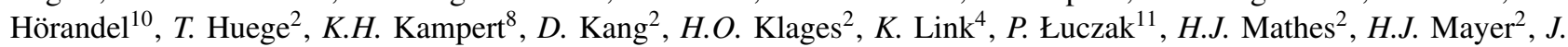

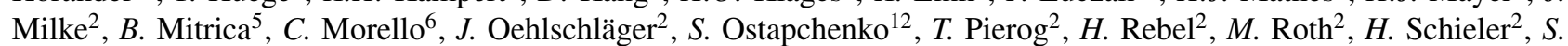

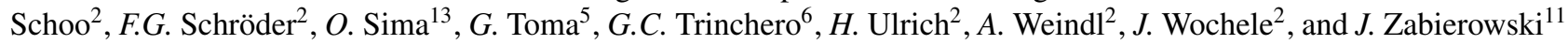 \\ ${ }^{1}$ Institute of Physics and Mathematics, Universidad Michoacana de San Nicolás de Hidalgo, Morelia, Mexico \\ ${ }^{2}$ Institut für Kernphysik, KIT - Karlsruher Institut für Technologie, Germany \\ ${ }^{3}$ Departimento di Fisica, Università degli Studi di Torino, Italy \\ ${ }^{4}$ Institut für Experimentelle Teilchenphysik, KIT - Karlsruher Institut für Technologie, Germany \\ ${ }^{5}$ Horia Hulubei National Institute of Physics and Nuclear Engineering, Bucharest, Romania \\ ${ }^{6}$ Osservatorio Astrofisico di Torino, INAF Torino, Italy \\ ${ }^{7}$ Universidade de São Paulo, Instituto de Física de São Carlos, Brasil \\ ${ }^{8}$ Fachbereich Physik, Universität Wuppertal, Germany \\ ${ }^{9}$ Department of Physics, Siegen University, Germany \\ ${ }^{10}$ Department of Astrophysics, Radboud University Nijmegen, The Netherlands \\ ${ }^{11}$ National Centre for Nuclear Research, Department of Astrophysics, Lodz, Poland \\ ${ }^{12}$ Frankfurt Institute for Advanced Studies (FIAS), Frankfurt am Main, Germany \\ ${ }^{13}$ Department of Physics, University of Bucharest, Bucharest, Romania \\ ${ }^{14}$ Now: Head of Division V at KIT - Karlsruher Institut für Technologie, Germany \\ ${ }^{15}$ Now at: Istituto Nazionale di Ricerca Metrologica, INRIM, Torino, Italy \\ ${ }^{16}$ Now at: DLR Oberpfaffenhofen, Germany \\ ${ }^{17}$ Now at: University of Duisburg-Essen, Duisburg, Germany \\ ${ }^{18}$ Spokesperson KASCADE-Grande
}

\begin{abstract}
In this work, we report measurements on the muon content $\left(E_{t h}>230 \mathrm{MeV}\right)$ of extensive air showers (EAS) induced by cosmic rays with primary energy from $10 \mathrm{PeV}$ up to $1 \mathrm{EeV}$ performed with the KASCADE-Grande experiment. The measurements are confronted with SIBYLL 2.3. The results are focused on the dependence of the total muon number and the lateral density distribution of muons in EAS on the zenith angle and the total number of charged particles in the shower. We also present updated results of a detailed study of the attenuation length of shower muons, which reveal a deviation between the measured data and the predictions of the post-LHC hadronic interaction models SIBYLL 2.3, QGSJET-II-04 and EPOS-LHC.
\end{abstract}

\section{Introduction}

At very high-energies, collisions of cosmic rays with the Earth's upper atmosphere produce an extensive air shower (EAS) of particles ( $\gamma^{\prime} s, e^{ \pm}$'s, muons, hadrons, etc.) in the forward direction, whose study can provide valuable information over the main properties of the primary particle. Unfortunately, the interpretation of the EAS data relies on the employment of hadronic interaction models, which are subject to theoretical and experimental uncertainties that may hamper composition studies of cosmicrays. Recently, a lot of progress has been done towards the reduction of such uncertainties motivated, in part, by the results of measurements at the LHC [1]. In this regard, several models were updated, for instance, SIBYLL

\footnotetext{
*e-mail: arteaga@ifm.umich.mx

**e-mail: drivera@ifm.umich.mx

***e-mail: andreas.haungs@kit.edu
}

2.3 [2], QGSJET-II-04 [3] and EPOS-LHC[4]. To check the reliability of such models at the energies relevant for EAS studies, the predictions of the models can be compared with data from air shower observatories. In this regard, the study of the shower muons becomes extremely useful, since they are sensitive to the hadronic interactions that occur in the early phases of the EAS development [5]. Hence, a failure of the models to describe the shower muon data may imply a problem in our phenomenological description of the hadronic physics of EAS. In this paper, we present preliminary results obtained from three tests of the above post-LHC models using shower muons measured with the KASCADE-Grande observatory.

KASCADE-Grande was a ground-based air-shower observatory dedicated to investigate the energy spectrum, the composition and the arrival direction of cosmic rays in the energy range from $10^{15}$ to $10^{18} \mathrm{eV}$ [6]. The instrument 
was located at the Karlsruhe Institute of Technology, Campus North (110 m a.s.1., $1022 \mathrm{~g} / \mathrm{cm}^{2}$ atmospheric depth) in Karlsruhe, Germany and took data from December 2003 up to November 2012. It consisted of several detector systems aimed to measure with high precision different components and properties of the EAS. One of the main systems of the observatory was the Grande array $\left(0.5 \mathrm{~km}^{2}\right.$ of area), which was composed of 37 scintillator detectors separated by a mean distance of $137 \mathrm{~m}$. The data collected with Grande was employed to estimate the shower core position at ground, the angle of incidence and the shower size or total number of charged particles (i.e. $e^{ \pm}$'s plus $\mu$ 's with $E_{t h}>3 \mathrm{MeV}$ for vertical incidence) of the event. Another important detector system of the observatory was the set of 192 shielded scintillator detectors from the KASCADE array $\left(200 \times 200 \mathrm{~m}^{2}\right)$, which provided local muon density measurements $\left(E_{t h}>230 \mathrm{MeV}\right)$ of the EAS and, hence, information about the total number of muons, $N_{\mu}$, in the shower.

\section{Experimental and simulated data}

The present studies were carried out using events collected from zenith angles $\theta<40^{\circ}$ and during the full data acquisition period of the experiment. In addition to reducing the effect of systematic uncertainties in the results, several selection cuts were applied to the data (see [7] for a detailed description). For example, to avoid border and punch-through effects, only events with cores located within the limits of a central area of $2.2 \times 10^{5} \mathrm{~m}^{2}$ inside the KASCADE-Grande array and within radial distances in the interval $R=[100 \mathrm{~m}, 600 \mathrm{~m}]$ were selected. After applying selection cuts, systematic uncertainties for $N_{c h}$ and $N_{\mu}$ were found to to be $\leq 15 \%$ and $\leq 20 \%$, respectively, in the region of maximum efficiency, i.e. $\log _{10}(E / \mathrm{GeV}) \geq$ $7.2 \pm 0.3$.

On the other hand, MC data sets were built for different combinations of the abovementioned post-LHC models and Fluka 2011.2 [8]. The former were used to describe the high-energy $\left(E_{h} \geq 200 \mathrm{GeV}\right)$ hadronic interactions in the EAS, while the latter, the corresponding low-energy regime. The production and development of the EAS were simulated with CORSIKA 7.5 [9] and the response of the detector to the passage of the shower, with a GEANT 3.21 [10] based program. MC events were generated for zenith angles $\theta<42^{\circ}$ and the energy interval from $E=10^{14} \mathrm{eV}$ to $3 \times 10^{18} \mathrm{eV}$ using an $E^{\gamma}$ primary spectrum with spectral index $\gamma=-2$. For the analysis, the MC events were weighted in order to simulate an $E^{-3}$ power-law spectrum. $\mathrm{MC}$ data samples were produced for five primary nuclei: $\mathrm{H}, \mathrm{He}, \mathrm{C}, \mathrm{Si}$ and $\mathrm{Fe}$, each of them with roughly the same number of events. Finally, all of them were processed with the same reconstruction algorithm employed with the experimental events [6] and were subject to the same cuts that were applied to the measured data.

\section{Results of the model tests}

\subsection{Muon radial density distributions}

The purpose of this analysis is to compare the predictions of SIBYLL 2.3 and the KASCADE-Grande measure- ments on the mean muon lateral distributions at the shower plane, $\rho_{\mu}(r)$, at different zenith angles and $\log _{10}\left(N_{c h}\right)$ intervals. In fig. 1, upper panel, the results for vertical EAS $\left(\theta=\left[0^{\circ}, 16.71^{\circ}\right]\right)$ and three distinct shower size ranges are presented. The mean $\rho_{\mu}(r)$ distributions were obtained at shower disk coordinates and were built neglecting corrections due to atmospheric absorption when projecting on the shower disk plane. From fig. 1, it is seen that the measured distributions are in general within the MC predictions for pure protons and iron nuclei. However, the shape of the predicted lateral density distributions seems to be flatter than the measured one. The results for inclined events will be analyzed elsewhere [7].

\subsection{The total muon number}

The aim of this study is to confront the measurements on $N_{\mu}$ as a function of the shower size (as a proxy of the primary energy) versus the expectations from SIBYL 2.3 for hydrogen and iron nuclei and different $\theta$ ranges. As a first step, for this analysis, the accuracy of $N_{\mu}$ was improved by correcting both the MC and measured $N_{\mu}$ data for systematic biases. This was achieved using a correction function calibrated with SIBYLL 2.3 predictions (see, e.g., [12] for a description of this function). This way, the final bias on $N_{\mu}$ became smaller than $8 \%$. The experimental results and the SIBYLL 2.3 predictions for the dependence of $N_{\mu}$ on $N_{c h}$ are presented in the lower panel of fig. 1 for three zenith angle intervals: $\left[0.0^{\circ}, 16.71^{\circ}\right],\left[23.99^{\circ}, 29.86^{\circ}\right]$ and $\left[35.09^{\circ}, 40^{\circ}\right]$. From this figure, in general, it is observed that the measured $N_{\mu}$ is in agreement with the predictions of the SIBYLL 2.3 model. However, there are a few exceptions at high-zenith angles and high shower sizes for two data points. The reason could be the poor statistics for the respective $\log _{10}\left(N_{c h}\right)$ bins or misreconstructed events that passed the selection cuts. The cause is under study.

Finally, it is noticed that fig. 1 also shows an increment towards heavier nuclei of the measured data for inclined EAS. This effect could be attributed to a mismatch between the predicted and the measured $\theta$-evolution of $N_{\mu}$ for EAS, which has been observed before when testing other hadronic interaction models [12]. This point is investigated in the analysis of the next subsection.

\subsection{The muon attenuation length}

In order to investigate the behaviour of the corrected $N_{\mu}$ with $\theta$ for air showers, the attenuation length of muons in the Earth's atmosphere, $\Lambda_{\mu}$, was investigated using the method described in [12]. This parameter is especially useful for cross-checking hadronic interaction models and is sensible to shower properties such as the inelasticity, spectrum and production of pions, among others. Recent analyses performed in [12] have shown that $\Lambda_{\mu}$, as predicted by QGSJET-II-2, QGSJET-II-04, EPOS-LHC and SIBYLL 2.1 [13], deviates from the measured value at KASCADE-Grande for the primary energy interval $E$ $\left[10^{16.3}, 10^{17}\right] \mathrm{eV}$ and zenitn angles $<40^{\circ}$. From the results of the last subsection it is suspected that also the prediction of SIBYLL 2.3 could deviate from the experimental 

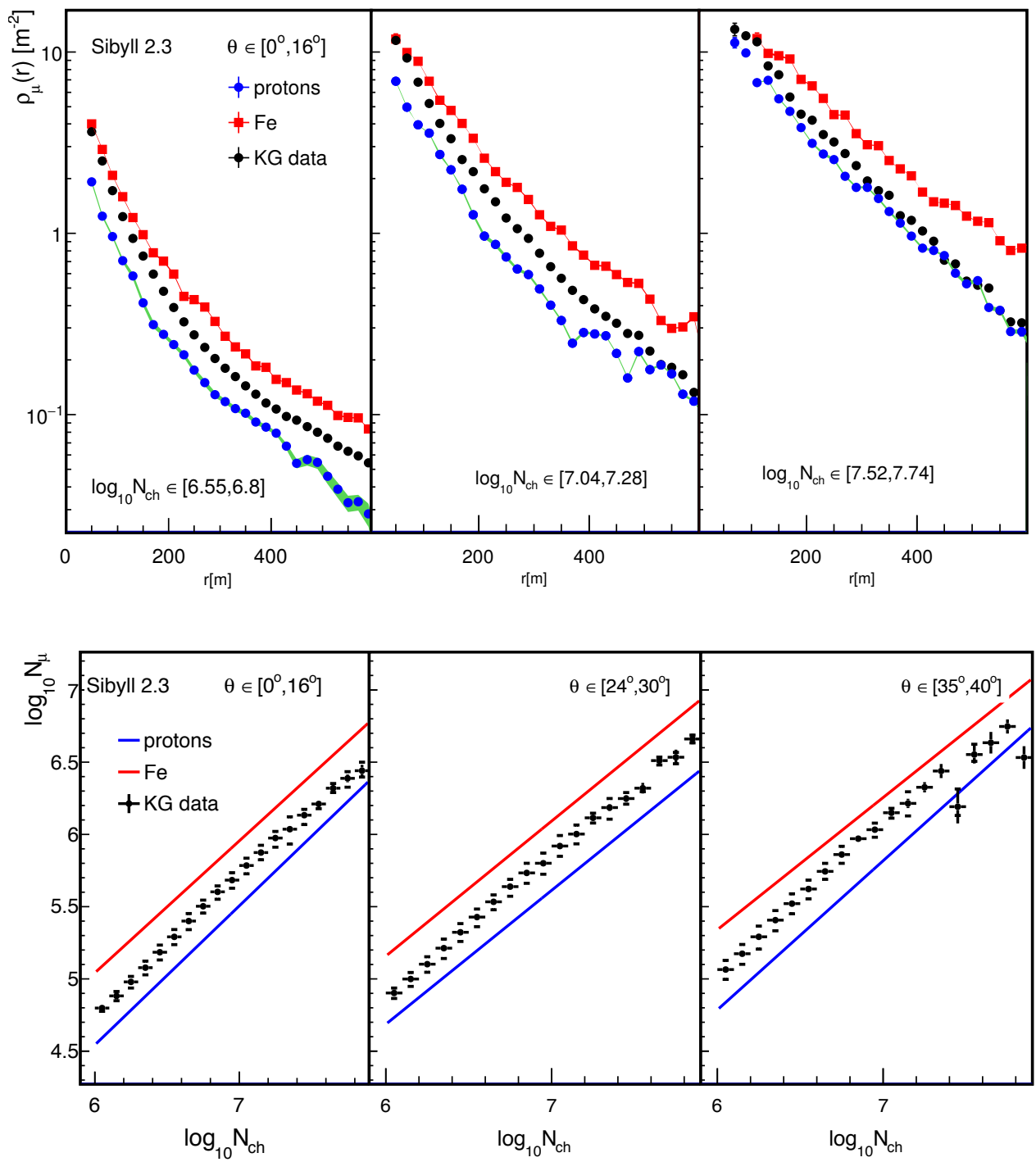

Figure 1. Upper panel: Mean muon lateral density distributions of EAS measured with KASCADE-Grande (points) compared with the predictions of SIBYLL 2.3 for iron (red upper lines) and proton (lower blue lines) primaries. Statistical error bars in measured data are smaller than the size of the markers. From MC simulations the energy scale of the above data spans the interval $E \sim\left[10^{7.2}, 10^{8.5}\right] \mathrm{GeV}$. Lower panel: Logarithm of the corrected muon size versus the logarithm of the total number of charged particles in air showers as measured with the KASCADE-Grande detector (black points). Measurements are confronted with predictions of SIBYLL 2.3 for iron nuclei (upper red lines) and protons (lower blue lines). Vertical error bars represent the systematic errors (corrected $N_{\mu}$ bias from reconstruction added in quadrature with an experimental bias that depends on the core position). Data is shown for the interval $N_{c h}=10^{6}-10^{7.9}$, which roughly corresponds to mean primary energies in the range $E \sim\left[10^{6.8}, 10^{9.1}\right] \mathrm{GeV}$ for the measured data. The primary energy for both panels was estimated event-by-event from both $N_{c h}$ and the corrected $N_{\mu}$ using a formula calibrated with SIBYLL 2.3 simulations (following the procedure of [11]).

result. To test this hypothesis an analysis like the one described in [12] was applied here. Also, the full data set of KASCADE-Grande was used to reduce the statistical error of the measurement in comparison with that presented in [12]. This also allowed to update the tests of $\Lambda_{\mu}$ presented in [12] for QGSJET-II-04 and EPOS-LHC.

Now, in order to reduce the systematic uncertainties of the expected $\Lambda_{\mu}$ due to the unknown cosmic ray composition a composition model derived from the data itself was applied to the MC data. The model was obtained by comparing, for each hadronic interaction model, a linear combination of templates for five mass groups $(\mathrm{H}, \mathrm{He}$,
$\mathrm{C}, \mathrm{Si}$ and Fe) with the measured $\log _{10}\left(N_{\mu}\right) / \log _{10}\left(N_{c h}\right)$ vs $\log _{10}\left(N_{\mu}\right)$ distribution. The MC templates for each mass group were weighted using double power-law formulas to model features of the individual spectra. The best parameters of these expressions were obtained by applying a loglikelihood fit using data with $\log _{10}\left(N_{\mu}\right) \geq 4$.8. Before weighting, the MC energy spectra resemble an $E^{-3}$ power-law function. It is worth mentioning that the measured and simulated $N_{\mu}$ data were corrected using the same correction function calibrated with the post-LHC model under consideration. An example of the result of the fit for SIBYLL 2.3 is presented in figure 2. We applied the re- 


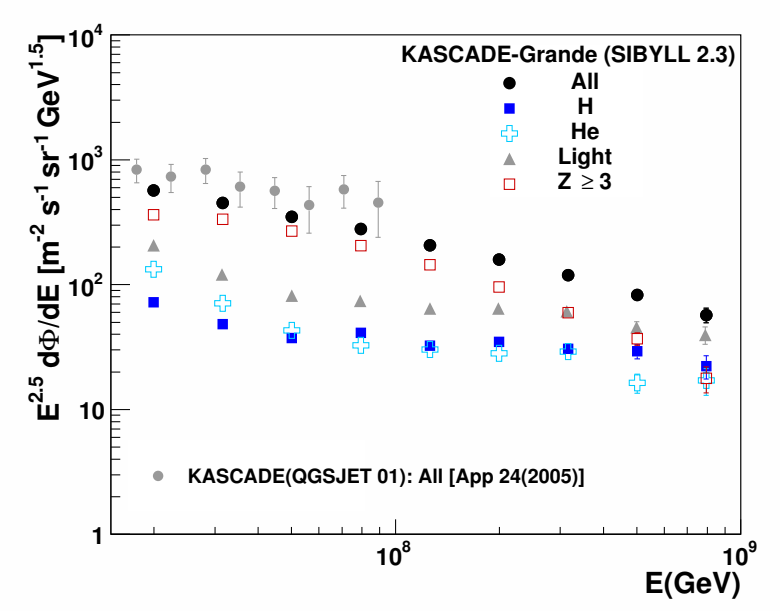

Figure 2. Results of the loglikelihood fit to the measured $\log _{10}\left(N_{\mu}\right) / \log _{10}\left(N_{c h}\right)$ distributions using SIBYLL 2.3 templates for five mass groups $(\mathrm{H}, \mathrm{He}, \mathrm{C}, \mathrm{Si}$ and $\mathrm{Fe}$ ) each of them following a double power-law energy spectrum. The fitted all-particle spectrum (black dots) and mass group spectra (H: solid blue squares, $\mathrm{He}$ : open sky blue circles and $\mathrm{C}+\mathrm{Si}+\mathrm{Fe}$ : open red squares) are shown for $\mathrm{E}=10 \mathrm{PeV}-1 \mathrm{EeV}$. The light component $(\mathrm{H}+\mathrm{He}$ : gray triangles) is also displayed.

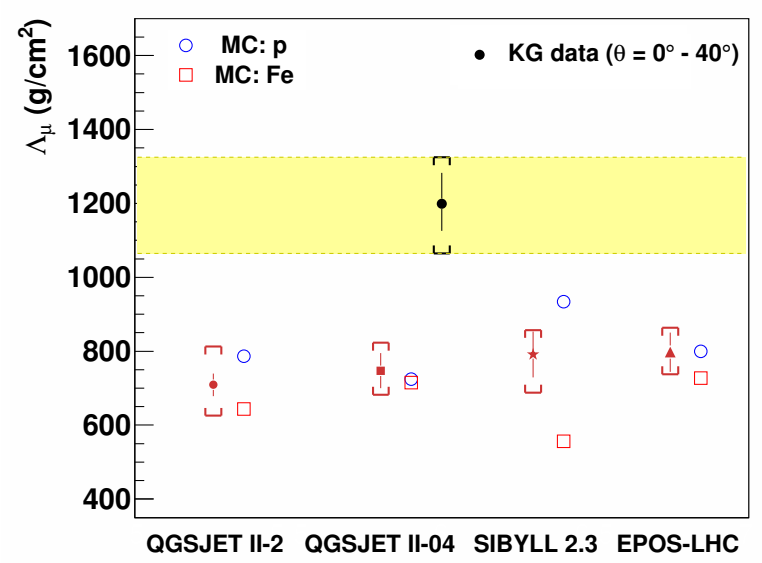

Figure 3. Muon attenuation length measured with the KASCADE-Grande experiment (upper point) compared with predictions (lower points) of QGSJET-II-02 (from [12]) and postLHC high-energy hadronic interaction models using our fitted composition models. Estimations for pure protons and iron nuclei (spectrum $E^{-3}$ ) are also shown (blue circles and red squares, respectively). The total uncertainty (statistical and systematic errors added in quadrature) are shown with squared brackets. Error bars represent statistical uncertainties (see [12] for a description of the uncertainties).

sulting weights to the respective MC simulations and use them to estimate $\Lambda_{\mu}$ for each model.

To obtain the muon attenuation length, we followed the procedure of [12]. In particular, we selected a sub-sample of data with radial distances, $R$, between $270 \mathrm{~m}$ and $440 \mathrm{~m}$ and within an area of $8 \times 10^{4} \mathrm{~m}^{2}$ located at the center of the KASCADE-Grande array, as they further reduce the systematic bias on $N_{\mu}$. Then, to obtain $\Lambda_{\mu}$, we applied the constant intensity cut (CIC) method [14] to the data as described in [12]. The same procedure was applied to the measured data sets and the MC models derived above. Results are presented in fig. 3. We observe that the predictions of the post-LHC hadronic interaction models for $\Lambda_{\mu}$ are in general below the measured value.

\section{Conclusions}

In this work, the SIBYLL 2.3 predictions on the muon content of EAS with primary energies around $E=10^{17} \mathrm{eV}$ have been tested using data from the KASCADE-Grande observatory. The results show that the measured data is in agreement with the predictions of the model. However, the actual muon lateral density distributions seems to be steeper than the model expectations. In addition the attenuation length of shower muons in the atmosphere was measured using the CIC method. It was found that the result is above the predictions of the post-LHC models SIBYLL 2.3, QGSJET-II-04 and EPOS-LHC.

\section{Acknowledgments}

The authors would like to thank the members of the engineering and technical staff of the KASCADE-Grande Collaboration, who contributed to the success of the experiment. The KASCADE-Grande experiment was supported in Germany by the BMBF and by the 'Helmholtz Alliance for Astroparticle Physics - HAP' funded by the Initiative and Networking Fund of the Helmholtz Association, by the MIUR and INAF of Italy, the Polish Ministry of Science and Higher Education, the Romanian Authority for Scientific Research UEFISCDI (PNII-IDEI grants 271/2011 and 17/2011), and the German-Mexican bilateral collaboration grants (DAAD-CONACYT 2009-2012, 2015-2016). J.C.A.V. acknowledges the partial support of CONACyT and the Coordinación de la Investigación Científica de la Universidad Michoacana.

\section{References}

[1] S. Ostapchenko, Proc. of the XXV ECRS, eConf C16-09-04.3 (2016).

[2] F. Riehn et al., Proc. of the 34th ICRC, PoS (ICRC2015) 558.

[3] S. Ostapchenko, Phys. Rev. D 83 (2011) 014018.

[4] T. Pierog et al., Phys. Rev. C 92, (2015) 034906.

[5] L. Cazon et al., Astrop. Phys. 2172 (2004); L. Cazon et al., Astrop. Phys. 23393 (2005).

[6] W.D. Apel et al., NIM A 620202 (2010).

[7] J.C. Arteaga-Velázquez et al., EPJ Web of Conferences 172, 07003 (2018).

[8] A.Fasso et al., Report CERN-2005-10,INFN/TC-05/11, SLAC-R773 (2005).

[9] D. Heck et al., Report FZKA 6019, Forschungszentrum Karlsruhe (1998).

[10] R. Brun, F. Carminati, CERN Program Library Long Writeup W5013 (1993).

[11] W.D. Apel et al., Astrop. Phys. 36183 (2012).

[12] W.D. Apel et al., Astrop. Phys. 95 (2017) 25-43

[13] E.J. Ahn et al., Phys. Rev D 80094003 (2009).

[14] J. Hersil et al., Phys. Rev. Lett. 622 (1961); D. M. Edge et al., J. Phys. A 61612 (1973). 